\section{Perfil de idosos com internação por quedas nos hospitais públicos de Niterói (RJ)}

\section{Profile of elderly admitted to public hospitals of Niterói $(R J)$ due to falls}

\section{Fátima de Lima Paula'}

Maria de Jesus Mendes da Fonseca'

Raquel de Vasconcellos Carvalhaes de Oliveira"

\section{Suely Rozenfeld"'}

' Escola Nacional de Saúde Pública da Fundação Oswaldo Cruz - FIOCRUZ.

"Laboratório de Epidemiologia Clínica do Instituto de Pesquisa Evandro Chagas

- IPEC.

III Departamento de Epidemiologia da Ensp da FIOCRUZ.

O estudo é resultado final de Dissertação de Mestrado no Programa de Pós-Graduação da Escola Nacional de Saúde Pública, subárea de Epidemiologia.

Correspondência: Fátima de Lima Paula. Escola Nacional de Saúde Pública . Rua Leopoldo BuIhões, $1480-8^{\circ}$ andar - Manguinhos - Rio de Janeiro. RJ CEP 21041-210. E-mail: fatima.lima.paula@ gmail.com

\section{Resumo}

Objetivo: Descrever as características comuns de idosos internados, por quedas, em hospitais públicos do município de Niterói - RJ. Métodos: Estudo seccional com 110 pacientes com 60 anos ou mais, nos hospitais públicos de Niterói, internados devido a fraturas pós-queda. Os dados foram coletados com um questionário multidimensional aplicado por meio de entrevista. Foram utilizadas a análise de correspondência múltipla e a análise de conglomerados para descrever as características dos entrevistados. Resultados: Os resultados apontaram quatro grupos. O primeiro grupo era composto de idosos com melhores condições físicas: não fraturaram o fêmur, eram mais independentes, saíam mais de casa antes da queda, tinham boa visão, permaneceram menos tempo internados e saíram do hospital com alta para casa. Outro grupo apresentou indivíduos em piores condições físicas: os menos independentes, os que ficaram mais tempo internados e os que saíram do hospital por óbito. Ainda foi apontado um grupo dos que fraturaram o fêmur, não tinham boa visão e dos que saíam de casa menos de uma vez por semana. O quarto grupo apontou os que saíam de casa uma a duas vezes por semana e os que ficaram internados de 11 a 30 dias. Conclusões: A análise de correspondência múltipla foi uma técnica útil para identificar subgrupos de pacientes com características comuns, o que fornece indícios para a criação de estratégias nos programas de prevenção de quedas.

Palavras chave: Análise de correspondência. Análise de conglomerados. Fraturas. Quedas. Idosos. Hospitalização. 


\section{Abstract}

Objective: to describe the common characteristics of elderly people admitted to public hospitals of Niterói (RJ) due to falls. Methods: Cross-sectional study with 110 elderly patients (60 years and more) admitted to public hospitals in Niteroi - RJ, with fractures after falls. Data were collected by a multidimensional questionnaire. Multiple correspondence and cluster analysis were used to describe the characteristics of this population. Results: The results indicated four groups. The first group was formed by individuals with better physical conditions who did not have a hip fracture, were more independent, used to go out more than twice a week, had good vision, had a short hospital stay, and were discharged to their homes. There was a group with worse conditions including those who were less independent, had a long hospital stay (more than 30 days), and died at the hospital. A third group was formed by those who had had a hip fracture, had poor vision, and used to go out less than once a week. The fourth group was comprised of those who stayed in the hospital from 11 to 30 days and used to go out once or twice a week. Conclusions: The multiple correspondence analysis proved to be a good technique to identify subgroups with common characteristics, which provides tools for the creation of strategies for fall prevention programs.

Keywords: Correspondence analysis. Cluster analysis. Fractures. Falls. Elderly. Hospitalization.

\section{Introdução}

O envelhecimento populacional é uma realidade mundial. Com o aumento da expectativa de vida e o envelhecimento populacional criou-se um desafio: proporcionar melhor qualidade de vida para aquele que envelhece. A Organização Mundial de Saúde determina que, em países em desenvolvimento, idoso é aquele com 60 anos ou mais. Tem-se observado que o que mais compromete a qualidade de vida do idoso é a perda de autonomia e independência, ou seja, a ausência de habilidade para desempenhar suas atividades ${ }^{1}$.

A queda vem sendo apontada como um dos problemas que podem limitar a independência e a autonomia do idoso. Por ser um evento multifatorial, o estudo do risco de cair é complexo. Assim, se um idoso apresenta vários fatores de risco terá uma chance maior de cair do que aquele que apresenta um único fator de risco. Por outro lado, se ele apresenta um alto grau de acometimento por apenas um fator, seu risco aumenta. Por isso, para avaliar a queda desse grupo etário de forma adequada há que se considerar seu caráter multifatorial ${ }^{2}$.

Idade, sexo, alterações de equilíbrio e da propriocepção, alterações visuais e vestibulares, uso de medicamentos, fraqueza muscular, déficit da capacidade funcional e fatores ambientais são fatores de risco de quedas de idosos apontados na literatura ${ }^{3,4}$. Segundo Tinetti e col. ${ }^{5}$, 30\% dos idosos caem pelo menos uma vez em um ano e o percentual de idosos que caem aumenta de $8 \%$ - naqueles sem fator de risco - para $78 \%$ nos que apresentam quatro ou mais fatores de risco.

Perracini e Ramos ${ }^{6}$ relatam que as quedas são frequentes e trazem consequências que alteram negativamente a vida dos idosos. Portanto, as quedas deveriam ser evitadas por meio de medidas preventivas que considerem comprometimento da capacidade funcional, visão deficiente e falta de estimulação cognitiva.

Tinetti $^{7}$ aponta que até 1985 a queda 
era considerada um evento natural não prevenível. Contudo, a partir de 1986, pesquisadores de todo o mundo contribuíram com a descrição das morbidades físicas e psicológicas associadas com a queda, o que fez com que se pudesse compreendê-la como fenômeno capaz de ser previsto e prevenido. O conhecimento das relações entre as quedas e os potenciais fatores ligados à intervenção podem beneficiar os idosos na prevenção de futuras quedas e suas consequências e manter, ou até melhorar, a qualidade de vida dos mesmos ${ }^{1}$.

O objetivo deste estudo foi descrever características comuns de idosos que sofreram quedas e foram internados nos hospitais do SUS de Niterói (RJ), utilizando a técnica de análise de correspondência.

\section{Métodos}

\section{População e fonte de informação}

Trata-se de um estudo descritivo realizado a partir de uma população de 145 idosos (60 anos ou mais) internados nas enfermarias de três hospitais públicos de Niterói (RJ), tendo como causa a queda. Foram realizadas entrevistas com questionário estruturado, durante o período de seis meses, de 14 de janeiro a 14 de julho de 2008.

A análise foi realizada com dados de 110 idosos. Oito foram excluídos, por não terem condições físicas ou mentais para responder o questionário; sete se recusaram a participar; onze não foram encontrados, em razão de transferência para outro hospital ou em virtude de alta; e nove por apresentarem valores nulos para as variáveis utilizadas nesta análise. Dessas perdas, $65,6 \%$ eram do sexo feminino, 58,0\% fraturaram o fêmur e a idade média era 73,8 anos.

As variáveis utilizadas para a análise de correspondência foram: fratura do fêmur ( $\operatorname{sim} /$ não); visão boa ( $\operatorname{sim} /$ não); grau de independência (índice de Katz $<17$, índice de Katz $\geq 17$ ); frequência com que saía de casa antes da queda $(<1$ vez por semana, 1 a 2 vezes por semana, 3 ou mais vezes por semana); motivo da saída do hospital (alta ou óbito) e tempo de internação (1 a 10 dias, 11 a 30 dias, 31 dias ou mais).

\section{Índice de Katz}

$\mathrm{O}$ índice de $\mathrm{Katz}^{8}$ é o índice de independência nas atividades de vida diária (AVD), desenvolvido por Sidney Katz para a avaliação funcional dos indivíduos em seis diferentes atividades: banhar-se, vestirse, transferir-se da cama para a cadeira e vice-versa, ir ao banheiro, ser continente e alimentar-se. No presente estudo, para as três opções de resposta sobre cada uma dessas atividades, foram atribuídos os seguintes escores: não recebe nenhuma assistência, 3 pontos; recebe assistência parcial, 2 pontos; não executa a atividade, 1 ponto. $O$ índice é construído pela soma dos escores, ou seja, com variação de 6 a 18 pontos. O índice de independência foi categorizado por meio de ponto de corte de 17 pontos, em dois grupos:

- Grupo dos mais independentes (índice $\geq 17$ pontos) - indivíduos que não necessitavam qualquer assistência em todas as atividades ou que precisavam de assistência parcial em apenas uma atividade.

- Grupo dos menos independentes (índice $<17$ pontos) - indivíduos que necessitavam assistência parcial ou total em mais de uma atividade.

\section{Análise estatística}

$\mathrm{Na}$ análise descritiva foram utilizados desvio padrão, média e mediana das variáveis contínuas (idade e tempo de internação); e frequências em números absolutos e percentuais das variáveis categóricas.

Para explorar relações conjuntas das características dos idosos foram utilizados os pacotes estatísticos ca e rgl do programa R 2.8.1 (www.r-project.org).

O estudo do conjunto dos fatores associados foi efetuado com análise de correspondência múltipla e análise de conglomerados. O objetivo dessa técnica foi redução da dimensionalidade dos sinais 
e sintomas relacionados à queda. Nesta redução procurou-se criar agrupamentos da amostra formados por indivíduos com características similares.

\section{Análise de correspondência e de conglomerados}

A análise de correspondência múltipla (ACM) é uma técnica de caráter exploratório utilizada para análise de dados categóricos de tabelas de contingência, com um grande número de variáveis, para encontrar dimensões que permitam posicionar as categorias de resposta em um mesmo sistema de eixos ${ }^{9-11}$. A característica da técnica é a visualização gráfica das relações entre as diversas categorias das variáveis, ao identificar grupos que possuem os mesmos fatores de risco e, portanto, maior proximidade geométrica. Pela análise de correspondência, podem ser interpretadas como associações as posições das categorias de cada variável no plano multidimensional.

Essa técnica não faz suposições sobre a distribuição dos dados, o que permite a investigação de diversos padrões de associação, inclusive não-lineares. Por outro lado, o método não permite inferências para outras amostras devido ao seu aspecto descritivo e à ausência de pressupostos. Para obter planos que representem a configuração das categorias das variáveis no espaço, calculase um conjunto de eixos fatoriais, cada um maximizando uma parcela da variabilidade dos dados ${ }^{9}$.

A inércia total foi a medida utilizada para definir a proporção da variabilidade explicada por cada dimensão. Quanto maior a inércia, maior a associação entre linha e coluna $^{12}$. A inércia total absoluta foi decomposta em inércias parciais para cada dimensão (inércias relativas), o que permite que se conheça quanto da inércia total está sendo explicada por uma dada dimensão derivada. A raiz quadrada da inércia corresponde a uma medida chamada autovalor, que sugere quanto das variâncias totais das medidas está sendo coberto pela dimensão ${ }^{13}$.

Na solução final só são mostrados os autovalores correspondentes ao método de extração conhecido como ajuste das inércias, que define o número de dimensões que melhor decompõe a inércia. $\mathrm{Na}$ interpretação dos dados foi fornecido o gráfico formado pelas coordenadas de cada categoria em cada dimensão, além das contribuições de cada variável por dimensão.

A análise de conglomerados tem como objetivo dividir os elementos de uma amostra em grupos, de forma que elementos pertencentes a um mesmo grupo sejam similares entre si, com respeito às variáveis (características) que neles foram medidas, $\mathrm{e}$ que elementos em grupos diferentes sejam heterogêneos em relação a estas mesmas características ${ }^{14}$. O método de aglomeração dos dados empregado foi o average, que permite, através da utilização das distâncias obtidas pela análise de correspondência, confirmar os grupos encontrados pela inspeção visual. A realização da análise de conglomerados com as coordenadas obtidas pela análise de correspondência busca trazer maior objetividade à interpretação gráfica da análise de correspondência.

\section{Aspectos Éticos}

Este projeto foi submetido ao Comitê de Ética em Pesquisa da Universidade Federal Fluminense, que o aprovou e o registrou sob o número 184/06. Para isso foram obtidas autorizações assinadas pelos diretores dos hospitais. O termo de consentimento foi assinado pelo paciente ou por seu acompanhante.

\section{Resultados}

Dos 110 idosos cujas informações foram utilizadas para a análise, $68,2 \%$ eram do sexo feminino. A idade variou de 60 a 94 anos (média 75,24 anos DP=8,86 anos), sendo $44,0 \%$ entre 70 a 79 anos. Observou-se a predominância de viúvos $(48,2 \%)$ e dos analfabetos e sem primário completo $(63,2 \%)$. O local das fraturas variou sendo, que $78,2 \%$ da população fraturaram membro inferior, $16,4 \%$ membro superior, $3,6 \%$ fraturaram 
ambas as regiões e 1,8\% fraturaram outras regiões (um fraturou a clavícula e outro o crânio). A hipertensão arterial foi a doença de maior prevalência (64,5\%). A maioria dos pacientes apresentava mais de um problema de saúde (dados não apresentados em tabela).

Na população do estudo, $64,6 \%$ referiram estar com a visão boa (incluindo o uso de óculos). Dos indivíduos internados, $69,1 \%$ fraturaram o fêmur. Desses $(n=76)$, $14,5 \%$ morreram.

O tempo de internação variou de 1 a 52 dias (mediana $=14$ dias, $\mathrm{IQR}=16$ dias). A frequência com que os participantes saíam de casa antes da queda variou bastante. Dos 110 idosos, 53,6\% saíam três ou mais vezes por semana, $31,8 \%$ saíam de casa menos de uma vez por semana, $14,6 \%$ saíam de 1 a 2 vezes por semana.

Entre os 27 indivíduos classificados como menos independentes, $25,9 \%$ morreram, $11,1 \%$ ficaram internados por até 10 dias e $40,7 \%$ permaneceram no hospital por mais de 30 dias. Entre os mais independentes ( $\mathrm{n}=83$ ), $4,8 \%$ morreram, $39,8 \%$ ficaram internados por até 10 dias e 9,6\% ficaram internados por mais de 30 dias.

A Tabela 1 apresenta os autovalores e o percentual acumulado da variabilidade explicada por cada eixo de dimensão. Verifica-se que as duas primeiras dimensões explicam $86,4 \%$ da variabilidade global (ou inércia total) dos dados, a primeira dimensão contribuindo com $85,0 \%$. Na Tabela 2 são fornecidas as contribuições absolutas e relativas de cada variável. Pode-se observar que as variáveis que mais contribuíram no primeiro eixo foram índice de Katz $<17$, não fraturar o fêmur, sair de casa $<1$ vez/ semana e tempo de internação de 1 a 10 dias. Já no segundo eixo, cuja contribuição foi pequena, a variável que mais contribuiu foi tempo de internação 11 a 30 dias.

A Figura 1 mostra quatro grupos de variáveis na análise das dimensões 1 e 2 . Esses grupos, selecionados a partir da inspeção visual do gráfico gerado pela análise de correspondência, foram denominados:

Grupo A - aqueles que não fraturaram o fêmur, os que tiveram tempo de internação de 1 a 10 dias, os que saíam de casa 3 dias ou mais por semana, os que tiveram alta, tinham boa visão e eram mais independentes;

Grupo B - os que tiveram tempo de internação de 11 a 30 dias e os que saíam de casa 1 a 2 vezes por semana;

Grupo C - os que saíam de casa menos de 1 vez por semana, os que fraturaram o fêmur e os que tinham visão ruim;

Grupo D - os que tiveram tempo de internação de 30 dias ou mais, os menos independentes e os que foram a óbito.

A Figura 2 apresenta o dendrograma formado pela análise de conglomerados, que confirma os grupos formados pela análise de correspondência. Esta figura mostra que, ao utilizar um corte de quatro grupos, foram confirmados os mesmos grupos de variáveis do gráfico de correspondência.

\section{Discussão}

A maioria da população estudada era composta por pessoas do sexo feminino, viúvos, com escolaridade até primário incompleto; a média da idade foi 75,6 anos. A hipertensão foi a doença de maior prevalência entre eles. Os dados dos pacientes dos três hospitais estudados foram analisa-

Tabela 1 - Autovalores, percentual de variância por cada dimensão e percentual cumulativo Table 1 - Eigenvalues, variance proportion for each dimension and cumulative percentage

\begin{tabular}{lccc}
\hline Autovalores & \multicolumn{3}{c}{ Dimensões } \\
\cline { 2 - 4 } & 1 & 2 & 3 \\
\hline Valores & 0,072 & 0,001 & 0,000 \\
$\%$ inércia parcial & 85,0 & 1,4 & 0,4 \\
$\%$ inércia total & 85,0 & 86,4 & 86,7 \\
\hline
\end{tabular}


Tabela 2 - Distribuição da contribuição absoluta e relativa da análise de correspondência para os dois primeiros eixos segundo as variáveis de quedas.

Table 2 - Distribution of the absolute and relative contribution of the correspondence analysis for the first two axes for fallrelated variables.

\begin{tabular}{lcccc}
\hline & \multicolumn{3}{c}{ Contribuição } \\
\cline { 2 - 4 } Variável & \multicolumn{2}{c}{ Eixo 1 } & \multicolumn{2}{c}{ Eixo 2 } \\
\cline { 2 - 4 } & Absoluta & Relativa & Absoluta & Relativa \\
\hline Fraturou o fêmur (sim) & 0,019 & 3,164 & 0,001 & 0,199 \\
Fraturou fêmur (não) & 0,042 & 3,164 & 0,003 & 0,199 \\
Saiu com óbito & 0,024 & 3,732 & 0,000 & 0,056 \\
Saiu com alta & 0,003 & 3,732 & 0,000 & 0,056 \\
Tempo de internação 1 a 10 & 0,029 & 3,147 & 0,005 & 0,522 \\
Tempo de internação 11 a 30 & 0,003 & 1,419 & 0,010 & 5,055 \\
Tempo de internação + 30 & 0,021 & 3,139 & 0,006 & 0,827 \\
Katz <17 & 0,047 & 3,118 & 0,002 & 0,166 \\
Katz 17 e 18 & 0,015 & 3,118 & 0,001 & 0,166 \\
Saía de casa < 1 vez/semana & 0,030 & 3,203 & 0,001 & 0,067 \\
Saía de casa 1 a 2 vezes/semana & 0,001 & 1,356 & 0,004 & 3,350 \\
Saía de casa $\geq 3$ vezes/semana & 0,024 & 3,424 & 0,000 & 0,021 \\
Visão boa (sim) & 0,003 & 3,201 & 0,001 & 0,570 \\
Visão Boa (não & 0,006 & 3,021 & 0,001 & 0,570 \\
\hline
\end{tabular}

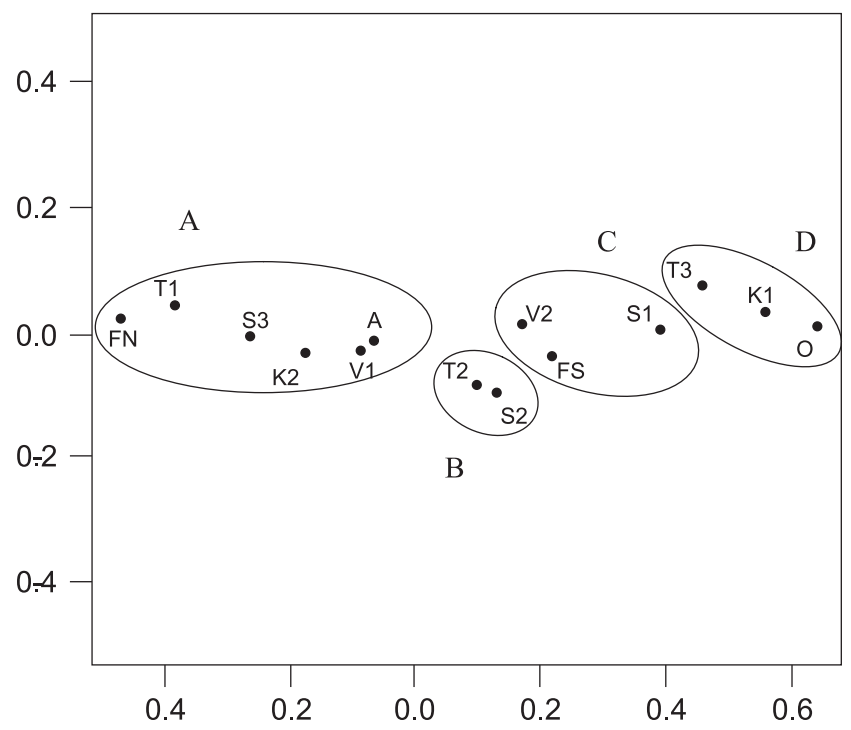

T1 - tempo de internação de 1 a 10 dias;T2 - tempo de internação de 11 a 30 dias;T3 - tempo de internação mais de 30 dias; S1 - saía de casa menos de 1 vez por semana; S2 - saía de casa de 1 a 2 vezes por semana; S3 - saía de casa de 3 a mais vezes por semana; FN -fraturou o fêmur (não); FS - fraturou o fêmur (sim); K1 - índice de Katz < 17; K2 - índice de Katz $\geq 17 ; \mathrm{V} 1$ - visão boa (sim); V2 - visão boa (não); A - alta; O - óbito.

T1 -1 to 10 days hospitalization;T2 -11 to 30 days hospitalization; T3 - more than 30 days hospitalization; S1 - going out less than once a week; S2 - going out once or twice a week; S3 - going out 3 or more times a week; FN - no hip fracture; FS - hip fracture; K1katz < 17;K2 - katz $\geq 17$; V1 - good vision (yes); V2 - good vision (no); A -discharge to home; O-died in hospital.

Figura 1 - Representação gráfica das duas primeiras dimensões da análise de correspondência. Figure 1 - Graphic representation of the first two dimensions of the correspondence analysis. 


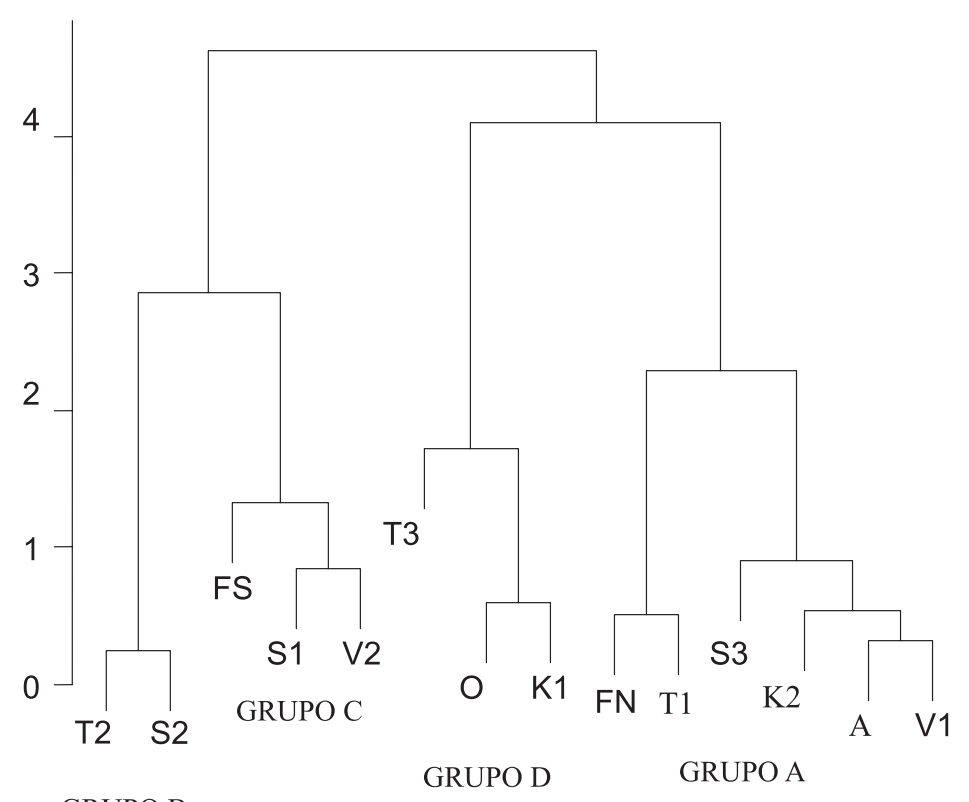

Figura 2 - Dendrograma das coordenadas das duas primeiras dimensões da análise de correspondência.

Figure 2 - Dendrogram of the coordinates of the first two dimensions of the correspondence analysis.

dos em conjunto, já que todos pertencem ao SUS e possivelmente recebem população semelhante. No presente estudo, o número de idosos internados por quedas (145) está muito próximo do encontrado no Datasus (141) no período de janeiro a julho de 2008.

A capacidade funcional parece ser importante para manter a qualidade de vida do idoso. Pode-se observar que, no grupo A, os indivíduos apresentavam melhores condições físicas: foram aqueles que não fraturaram o fêmur, os que permaneceram menos tempo internados, os que saíam de casa com maior frequência, os que tiveram alta, tinham boa visão e eram mais independentes. $\mathrm{O}$ resultado sugere que os mais independentes e mais ativos tiveram menos tempo internados e menor taxa de mortalidade. O grupo D foi formado por aqueles com maior tempo de internação, os menos independentes e os que foram a óbito. Com a idade, o indivíduo tende a diminuir suas atividades físicas, o que pode acarretar em diminuição da capacidade funcional. Embora o tempo de internação dependa de muitas outras variáveis, o agrupamento dos menos independentes com os que ficaram mais tempo internados pode sugerir que o idoso mais independente apresente condições físicas melhores para se recuperar quando hospitalizado por quedas. Segundo Siqueira et al. ${ }^{15}$, a capacidade funcional é um importante marcador de saúde em idosos hospitalizados. Aqueles que apresentam menores dificuldades nas atividades de vida diárias referidas na internação apresentam melhora funcional durante a internação. Os autores também verificaram associação direta e significativa entre comprometimentos funcionais e tempo de hospitalização $(\mathrm{p}=0,004)$.

O grupo C aproximou aqueles que fraturaram o fêmur, os que saíam de casa menos vezes por semana e os que relataram não estar com boa visão. É possível que indivíduos com déficit visual tendam a sair menos de casa. Segundo Veras et al. ${ }^{16}$, grande parte das alterações visuais dos idosos não chega a ser diagnosticada e acaba por agravar outras incapacidades funcionais. Assim, a menor velocidade para a leitura e a diminuição da abrangência do campo visual levam à perda de interesse por uma atividade prazerosa, 
acabando por favorecer a depressão e o isolamento social.

O grupo B dos que ficaram internados de 11 a 30 dias e dos que saíam de casa 1 a 2 vezes por semana não apresentou nenhuma relação relevante, não contribuindo para o perfil dos idosos dessa população.

Não foi encontrado na literatura estudo que tivesse utilizado a AC para verificar as relações conjuntas entre variáveis associadas a quedas. Uma limitação desse estudo é que a técnica utilizada não permite fazer inferência dos achados; mesmo assim, ela pareceu ser ferramenta útil para o estudo dessas relações e para traçar o perfil de subgrupos dessa população, alguns dos quais devem ser alvo prioritário das ações de controle do agravo.

O estudo das características comuns entre os idosos que são internados por quedas é importante para que se possa traçar um programa de prevenção, pois alguns destes fatores são possíveis de serem prevenidos.
Estudos ${ }^{17,18}$ apontam programas de prevenção que não só diminuem o números de quedas, mas também melhoram a qualidade de vida do idoso.

No Brasil ainda são poucos os programas que focam a prevenção de quedas, diferentemente do que ocorre em países como Canadá, Austrália, França e Estados Unidos ${ }^{19}$. Estudos sobre as características do idoso que cai são importantes para que sejam traçados programas deste tipo.

Espera-se que as características comuns encontradas neste estudo possam apontar direções para o planejamento de estratégias de programas de prevenção de quedas de idosos e fomentar mais estudos a partir das suposições levantadas. Estudos futuros, que observem grupos de idosos que caíram ou não, serão úteis para se ampliar o conhecimento dos fatores associados ao risco de quedas, assim como avaliar as relações conjuntas desses fatores entre os grupos.

\section{Referências}

1. Ramos LR. Fatores determinantes do envelhecimento saudável em idosos residentes em centro urbano. Projeto Epidoso, São Paulo. Cad Saúde Pública 2003; 19(3): 793-7.

2. Ishizuka MA. Avaliação e comparação dos fatores intrínsecos dos riscos de quedas em idosos com diferentes estados funcionais [dissertação de mestrado]. Campinas: Faculdade de Educação da Unicamp; 2003.

3. Perracini MR. Prevenção e Manejo de quedas no Idoso. PEQUI - Portal Equilíbrio e Quedas em idosos. Disponível em http://pequi.incubadora.fapesp.br/ portal/quedas. [Acessado em 7 de janeiro de 2009]

4. Downtown J. Falls in the Elderly. In: Brocklehurst JC, Tallis RC, Fillit HM. Textbook of Geriatric Medicine and Gerontology. 4ed Churchill Livingstone; 1992; p. 317-24.

5. Tinetti ME, Speechly M, Ginter SF. Risk factor for falls among elderly persons living in the community. $N$ Engl $J$ Med 1988; 319(26): 1701-7.

6. Perracini MR, Ramos LR. Fatores associados a quedas em uma coorte de idosos residentes na comunidade. Rev Saúde Pública 2002; 36(6): 93-9.

7. Tinetti ME. Where is the vision for fall prevention? JAGS 49(5): 676-77.
8. Katz S, Ford AB, Moskowitz RW, Jackson BA, Jaffe MW Studies of illness in the aged: The index of ADL: a standardized measure of biological and psychosocial function. JAMA 1963; 185(12): 914-9.

9. Mota JC, Vasconcelos AGG, Assis SG. Análise de correspondência como estratégia para descrição do perfil da mulher vítima do parceiro atendida em serviço especializado. Ciência \& Saúde Coletiva 2007; 12(3): 799809.

10. Mota JC, Vasconcelos AGG, Assis SG. Correspondence analysis: a method for classifying similar patterns of violence against women. Cad Saúde Pública 2008; 24(6): 1397-406.

11. Oliveira RVC, Silva AM, Assis SG, Santos NC. Análise de correspondência múltipla e análise de cluster na redução de dimensionalidade de indicadores de eventos de vida. Rev Bras Estatística 2006; 67: 95-116.

12. Greenacre, M. J., Practical correspondence analysis. In: Looking at Multivariate Data. New York: J. Wiley \& Sons; 1981.

13. Clausen SE. Applied correspondence analysis: an introduction. Quantitative application in the social sciences. Thousand Oaks, CA: Sage University Paper Series; 1988. 
14. Mingoti, S. A. Análise de Dados Através de Métodos de Estatística Multivariada: uma abordagem aplicada. Belo Horizonte: Editora UFMG; 2005.

15. Siqueira AB, Cordeiro RC, Perracini MR, Ramos LR. Impacto funcional da internação de pacientes idosos. Rev Saúde Pública 2004; 38(5): 687-94.

16. Veras RP, Caldas CP, Coelho FP, Sanchez MA. Promovendo a saúde e prevenindo a dependência: identificando indicadores de fragilidade em idosos independentes. Rev Bras Geriatr Gerontol 2007; 10(3): 355-70.
17. Li F, Harmer P, Fisher KJ, McAuley E, Chaumeton N, Eckstrom E, Wilson NL. Tai Chi and fall reduction in older adults: a randomized controlled trial. J Geront Med Sci 2005; 60A(2): 187-94.

18. Day L, Fildes B, Gordon I, Fitzharris, Flamer H, Lord S. Randomised factorial trial of fall prevention among older people living in their own homes. Br Med J 2002; 325(7356): 128-33.

19. Preventing Falls: What Works. A CDC Compendium of Effective Community-based Interventions from Around the World. National Center for Injury Prevention and Control. Atlanta, Georgia; 2008.

Recebido em: 14/12/09

Versão final reapresentada em: 08/07/10

Aprovado em: 23/07/10 